Nervenarzt 2015 $\cdot 86: 523-524$

DOI 10.1007/s00115-015-4312-y

Online publiziert: 6. Mai 2015

(c) Springer-Verlag Berlin Heidelberg 2015

\title{
I. Hauth
}

Alexianer St. Joseph-Krankenhaus, Klinik für Psychiatrie, Psychotherapie und Psychosomatik, Berlin

\section{Strukturqualität und Behandlungsleitlinien in der stationären Versorgung}

sentliches Qualitätsmerkmal der Behandlung in Kliniken für Psychiatrie und Psychotherapie. ren Schutz des Gesetzes ( $\$ 27$ SGB V) [1] Diese gesetzliche Bestimmung beinhaltet insbesondere, dass psychisch Kranke ein besonderes Recht auf die erforderliche Diagnostik, eine wirksame Therapie und eine Versorgung in allen Behandlungssektoren haben. Besondere Erfordernisse ergeben sich dabei für psychisch Kranke bei hoher Krankheitsausprägung und Akuität. Dabei ist der Krankenhaussektor besonders betroffen.

Vor allem bei ausgeprägten psychischen Störungen sind die persönliche Kompetenz der Therapeuten und deren zeitlicher Einsatz gefragt. Medikamentöse oder andere, weniger zeitintensive apparative Prozeduren (wie die „repetitive transcranial magnetic stimulation“ oder Elektrokrampftherapie) können das therapeutische Gespräch und die persönliche, kompetente Zuwendung nicht ersetzen, wie wichtig diese biologischen Verfahren im Therapiegesamtplan auch sind. Wesentlich sind und bleiben unterstützende Gespräche, Psychotherapie und psychosoziale Interventionen und ausreichend Zeit, um tragfähige Beziehungen zu Patientinnen und Patienten aufzubauen. Psychotherapie und Soziotherapie sind wirksam erprobte Prozeduren, sie sind aber zeitlich nicht komprimierbar.

Eine weitere Besonderheit unseres Faches sind Vorhalteleistungen für schwer psychisch Kranke: Das betrifft die sog. Pflichtversorgung, die 24-stündige Aufnahmebereitschaft sowie auch im Verlauf der stationären Behandlung die 1:1Betreuung und Intensivbehandlung. Die Bereitstellung kompetenter und ausreichender Personalressourcen ist ein we-

\section{》) Selbst eine 100\%-Psych-PV- Ausfinanzierung genügt nicht den heutigen Anforderungen}

Anfang der 1990er Jahre hat die Personalverordnung Psychiatrie (Psych-PV) der gesetzlichen Bestimmung zum Schutz psychisch Kranker Rechnung getragen, indem sie auf der Grundlage einer (für diese Zeit) optimalen stationären Versorgungsstruktur den therapeutischen Stellenbedarf ermittelte, der als personelle Mindestausstattung gilt (Beitrag Klimke et al.). Die Psych-PV definiert somit eine normative Struktur auf Grundlage eines früher gültigen „state of the art". Seit der Einführung der Psych-PV haben sich die therapeutischen, gesetzlichen und die ethischen Rahmenbedingungen grundlegend verändert. Sie wurde initial voll ausfinanziert, aber im Laufe der folgenden Jahrzehnte durch Tarifsteigerungen ausgehöhlt. Die Psych-PV hat die Qualität der stationären Versorgung psychisch Kranker enorm verbessert. Aber selbst eine 100\%-Psych-PV-Ausfinanzierung kann den heutigen Qualitätsanforderungen nicht genügen.

Durch die Wirksamkeitsnachweise für störungsspezifische Psychotherapie und Soziotherapie ist ein erheblicher Therapiefortschritt zu verzeichnen! Während vor 20 bis 30 Jahren therapeutische Wirksamkeit vorzugsweise auf Psychopharmaka begrenzt war, haben sich mittlerweile psychotherapeutische Verfahren als mindestens gleich wirksam erwiesen. Es gibt keine psychische Erkrankung, in der nicht störungsspezifische Psychotherapieverfahren bzw. (im Fall der Demenz psychosoziale Interventionen) mindestens gleichrangig neben medikamentösen Therapien stehen. Die Behandlungsleitlinien in Deutschland, aber auch in allen anderen Ländern, geben daher die Kombination aus medikamentöser Behandlung und Psychotherapie als klare, alternativlose Empfehlung. Die Kombinationstherapie ist in jedem Fall wirksamer als eine alleinige Pharmakotherapie. Diese innovativen und in ihrer Wirksamkeit abgesicherten Therapieverfahren sind nur durch zeitliche Leistungen qualifizierten Personals zu erbringen. Die durch die Psych-PV bereitgestellten Ressourcen sind diesen heutigen Qualitätsanforderungen nicht mehr gewachsen, wie in den Beiträgen von Norman et al. und Berger et al. (s. unten) gezeigt wird. Vielmehr bedarf es einer Ausweitung der personellen Strukturanforderungen. Um hier hinlänglich die Qualität zu garantieren, sind also angepasste neue normative Personal- und Strukturkriterien nötig, die auch gegenzufinanzieren wären. Anders kann das anfangs zitierte Gebot, dass psychisch Kranke den besonderen Schutz des Gesetzes genießen, nicht erfüllt werden.

Die ethischen Rahmenbedingungen der Behandlung psychisch Kranker haben sich in den vergangenen 10 Jahren substanziell verändert. Der Respekt vor der Autonomie des Patienten und die daraus resultierenden Erfordernisse der Aufklärung über Behandlungsprozeduren sowie das intensive Bemühen um die Vermeidung von Zwangsmaßnahmen sind heu- 
te ethische Gebote. Diese Entwicklung ist vor allem bei psychischen Störungen relevant, denn besonders diese Erkrankungen können die Autonomie der Patienten bedrohen. „Fremdgefährdungen“ als Folge psychischer Erkrankungen erfordern z. B. eine besonders zeitintensive Zuwendung von Ärzten und Pflegern, um Einvernehmen $\mathrm{zu}$ erreichen und Zwangsmaßnahmen zu vermeiden. Eine besondere Nachdrücklichkeit erhielt diese Entwicklung durch die UN-Behindertenrechtskonvention, die für psychisch Kranke von hoher Relevanz ist [1]. Diese veränderten ethischen Rahmenbedingungen erfordern einen deutlich höheren Personalbedarf als es durch die Psych-PV vorgegeben war. Diese ethischen Erfordernisse können nur durch eine entsprechende Strukturqualität eingehalten werden.

Das neu einzuführende Krankenhausentgeltsystem (PEPP, pauschalierendes Entgeltsystem in Psychiatrie und Psychosomatik) hat sich eigentlich all diesen neuen Herausforderungen zu stellen. Es orientiert sich aber ausschließlich an $\mathrm{Pa}$ tientenmerkmalen und definierten therapeutischen Prozessen. Eine normative Struktur, die für Patienten optimale Behandlungsbedingungen schafft, ist damit nicht mehr gesichert. Um den aufgezeigten Entwicklungen zu genügen, ist eine Aufstockung der Finanzierung eigentlich unausweichlich. Diese ist aber nicht vorgesehen. Vielmehr setzt das neue Entgeltsystem auf einer Basis der Personalstruktur auf, die im Hinblick auf die evidenzbasierten Leitlinien nicht ausreichend ist. Damit stellt dieses geplante Entgeltsystem die Möglichkeit einer hinlänglich qualitätsorientierten stationären Versorgung psychisch Kranker infrage.

Das Psych-Entgeltgesetz sieht die Absicherung einer hinlänglichen Strukturqualität vor. Mit dieser Entwicklung ist der Gemeinsame Bundesausschuss (G-BA) beauftragt worden. Offen bleiben allerdings der verpflichtende Charakter der zu entwickelnden Kriterien und deren finanzielle Absicherung. Die wissenschaftlich geleitete Entwicklung von Kriterien für eine zukünftige Strukturqualität sollte dennoch als Chance begriffen werden.

Dabei ergeben sich zwei Erfordernisse:
1. eine für die heutige Zeit gültige Strukturqualität zu definieren und

2. deren Gegenfinanzierung zu ermöglichen.

Eine Gerechtigkeitslücke und erhebliche Risiken für die Qualität der künftigen stationären Versorgung psychisch Kranker ergeben sich jedenfalls dann, wenn der in unserem Fach erreichte medizinische Fortschritt psychisch Kranken vorenthalten wird.

Die drei Arbeiten zu diesem Schwerpunktthema in Der Nervenarzt zeigen, dass die Durchsetzung dieser Ziele nicht ohne zusätzliche Finanzmittel und/oder einer Strukturreform in der Versorgung psychisch Kranker zu leisten ist. Eingangs skizziert die Arbeit von Klimke et al. Grundsätze der gegenwärtigen und zukünftigen Strukturqualität in Kliniken für Psychiatrie und Psychotherapie aus verschiedenen Perspektiven (Patienten, Kliniken, Kostenträger, Politiker). Dabei werden vor allem auch die Gefährdungen beim Verlust einer gegenfinanzierten normativen Strukturqualität aufgezeigt.

Die beiden anderen Arbeiten berichten in empirischen Analysen über den Personalaufwand für eine leitliniengerechte Behandlung in einer Gruppe von Universitätskliniken. Gemeinsam ist diesen Kliniken die erfolgreiche Implementierung aller störungsspezifischen S3-Leitlinien. Norman et al. ermitteln in diesen Kliniken den ärztlich-psychologischen Personalbedarf. Sie vergleichen diesen ermittelten Bedarf sowohl mit den von der Psych-PV bereitgestellten Personalmitteln als auch mit dem bisher nur in Annäherung abschätzbaren, dafür bereitgestellten Ressourcen im PEPP-System. Das Ergebnis belegt klar: Beide Finanzierungs- bzw. Entgeltsysteme sind nicht geeignet, eine leitliniengerechte stationäre Behandlung zu ermöglichen.

Berger et al. ermittelten den Personalbedarf aller Berufsgruppen während einer fünfwöchigen Behandlung bei unipolarer Depression mit einem den Leitlinienempfehlungen entlehnten Wochenplan (je 280 min Gruppen- oder Einzeltherapie und klinische Basisbetreuung). Dieses Therapieangebot ist nach Metaanalysen für einen hinreichend guten, durchschnittlichen Therapieerfolg nötig.
Diesem Wert stehen nur 72 min Psychotherapie pro Woche nach Psych-PV bei 100\%-Umsetzung gegenüber. Die Autoren erwägen, diese Deckungslücke durch eine teilweise Flexibilisierung der Sektorgrenzen zwischen "stationär“, „tagesklinisch“ und „ambulant“ zu schließen.

Die Versorgungsforschung in unserem Fach ist also besonders gefordert, Rahmenbedingungen für eine ausreichende, an einer gegenwärtigen wissenschaftlichen Evidenzlage für wirksame Behandlungen orientierten Struktur für die Versorgung psychisch Kranker zu entwickeln und zwar auf allen Versorgungsebenen - stationär, teilstationär sowie ambulant - und für alle therapeutisch tätigen Berufsgruppen. Denn nur auf einer solchen Grundlage wird es möglich sein, dem an dieser Stelle aktiven untergesetzlichen Normgeber (der G-BA) die Mittel an die Hand zu geben, eine qualitätsgesicherte Versorgung psychisch Kranker zu gewährleisten. In den Folgeheften von Der Nervenarzt werden einige weitere Arbeiten diesen Themen gewidmet sein.

\section{Korrespondenzadresse}

\section{Dr. I. Hauth}

Alexianer St. Joseph-Krankenhaus, Klinik für Psychiatrie, Psychotherapie und Psychosomatik Gartenstraße 1, 13088 Berlin

i.hauth@alexius.de

Interessenkonflikt. I. Hauth gibt an, dass kein Interessenkonflikt besteht.

\section{Literatur}

1. Beauftragte der Bundesregierung für die Belange behinderter Menschen (Hrsg) (2014) Die UNBehindertenrechtskonvention. Übereinkommen über die Rechte von Menschen mit Behinderungen. Stand Oktober 2014. BMAS, Bonn 